\title{
A closer look at chaotic advection in the stratosphere: part II: statistical diagnostics
}

Article

Published Version

Ngan, K. and Shepherd, T. G. (1999) A closer look at chaotic advection in the stratosphere: part II: statistical diagnostics. Journal of the Atmospheric Sciences, 56 (24). pp. 4153-4166. ISSN 1520-0469 doi: https://doi.org/10.1175/15200469(1999)056<4153:ACLACA>2.0.CO;2 Available at https://centaur.reading.ac.uk/32856/

It is advisable to refer to the publisher's version if you intend to cite from the work. See Guidance on citing.

Published version at: http://dx.doi.org/10.1175/1520-0469(1999)056<4153:ACLACA>2.0.CO;2

To link to this article DOI: http://dx.doi.org/10.1175/1520-

0469(1999)056<4153:ACLACA>2.0.CO;2

Publisher: American Meteorological Society

All outputs in CentAUR are protected by Intellectual Property Rights law, including copyright law. Copyright and IPR is retained by the creators or other copyright holders. Terms and conditions for use of this material are defined in the End User Agreement.

www.reading.ac.uk/centaur 
Central Archive at the University of Reading

Reading's research outputs online 


\title{
A Closer Look at Chaotic Advection in the Stratosphere. Part II: Statistical Diagnostics
}

\author{
Keith NGan* AND Theodore G. ShePherd \\ Department of Physics, University of Toronto, Toronto, Ontario, Canada
}

(Manuscript received 17 September 1997, in final form 22 February 1999)

\begin{abstract}
Statistical diagnostics of mixing and transport are computed for a numerical model of forced shallow-water flow on the sphere and a middle-atmosphere general circulation model. In particular, particle dispersion statistics, transport fluxes, Liapunov exponents (probability density functions and ensemble averages), and tracer concentration statistics are considered. It is shown that the behavior of the diagnostics is in accord with that of kinematic chaotic advection models so long as stochasticity is sufficiently weak. Comparisons with random-strain theory are made.
\end{abstract}

\section{Introduction}

In the first part of this study, Ngan and Shepherd (1999, hereafter Part I), it was shown that chaotic advection occurs in both a shallow-water model of the winter stratosphere and a middle-atmosphere general circulation model. Specifically, the "cat's eye" structure in the surf zone organizes mixing and transport in a manner akin to the heteroclinic structure of a temporally aperiodic chaotic system; consequently velocity derivatives decorrelate along particle trajectories rather than at fixed spatial locations. This behavior is robust, and is consistent with previous studies of stratospheric mixing and transport, as well as with analytical and numerical results for Rossby wave critical layers.

The analysis of Part I was largely qualitative, the primary objective of that paper being to clarify the notion of chaotic advection in the stratosphere and determine conditions under which it may occur. It is the objective of the present paper to characterize chaotic advection in the shallow-water and middle-atmosphere models by computing a number of representative statistical diagnostics.

Since there does not exist a single diagnostic by which chaotic advection can be fully characterized, such an approach cannot establish unequivocally the "definitive" features of chaotic advection in the stratosphere,

\footnotetext{
* Current affiliation: Department of the Geophysical Sciences, University of Chicago, Chicago, Illinois.
}

Corresponding author address: Dr. Keith Ngan, Department of Geophysical Sciences, University of Chicago, 5734 S. Ellis Avenue, Chicago, IL 60637.

E-mail: kngan@midway.chicago.edu but it is, nonetheless, useful from both a practical and theoretical perspective. Practically, it enables one to make concrete many of the qualitative results of Part I (e.g., the shallow-water parameter dependences and the relative insensitivity of the Lagrangian correlation time to the forcing amplitude). Furthermore, as we consider diagnostics that have not been used much, if at all, in the stratospheric context-most notably the probability distribution of Liapunov exponents-the accompanying results also provide some insight into stratospheric mixing and transport. Theoretically, it is important to understand how chaotic advection in the stratosphere relates to chaotic advection in dynamical systems and to random-strain theory. While there are obvious analogies with the shallow-water and middle-atmosphere models, as noted in Part I, so too are there many differences. With respect to random-strain theory, for instance, the condition that all the velocity derivatives be random functions with fixed correlation time would not appear to be satisfied in the more complex models.

The statistical diagnostics analyzed in this paper may be usefully divided into transport and mixing diagnostics. As with Part I, the primary focus is on the shallowwater parameter dependence: the transport diagnostics, which characterize large-scale tracer movement, consist of particle dispersion statistics (section 2) and transport fluxes (section 3); the mixing diagnostics, which characterize the evolution of small-scale tracer structure (roughly speaking, stretching and homogenization), consist of Liapunov exponents (probability distribution functions and ensemble averages in particular; section 4), and tracer concentration statistics (section 5). Some calculations with isentropic winds from the Canadian Middle Atmosphere Model (CMAM) are described in section 6. Throughout the paper, the role of the largescale velocity field - the "organizing structure" - is em- 
phasized, particularly in relation to the theoretical results. Details on the requisite particle trajectory calculations, as well as on the formulation of the models, may be found in Part I.

\section{Dispersion statistics}

The zonal and meridional mean-square dispersion are given by

$$
\sigma_{x}^{2}=\left\langle(\lambda-\langle\lambda\rangle)^{2}\right\rangle, \quad \sigma_{y}^{2}=\left\langle(\phi-\langle\phi\rangle)^{2}\right\rangle,
$$

where $\lambda$ and $\phi$ are the longitude and latitude in radians; the angle brackets denote the ensemble average

$$
\langle\cdot\rangle=\sum_{i=1}^{N}(\cdot) \cos \phi_{i},
$$

$N$ being the number of particles. ${ }^{1}$ Roughly speaking, $\sigma_{y}^{2}$ characterizes the spatial extent of transport; $\sigma_{x}^{2}$ serves as a test for anomalous diffusion.

Unless stated otherwise the initial particle distribution consists of approximately 70000 particles uniformly spaced from $15.5^{\circ}$ to $52.5^{\circ} \mathrm{N}$ at $0.5^{\circ}$ intervals, and from $0.2^{\circ}$ to $359.8^{\circ} \mathrm{E}$ at $0.32^{\circ}$ intervals.

\section{a. Shallow-water parameter dependence}

In deterministic chaotic systems, the variation of $\sigma_{x}^{2}$ and $\sigma_{y}^{2}$ with the amplitude of the perturbation reflects changes in the underlying phase-space structure. We now consider whether there is similar behavior for the shallow-water system. Based on the results of Part I it is expected that there should be a close correspondence between the heteroclinic structure of a chaotic system and the organizing structure of the shallow-water system.

We begin with stationary forcing. Figure 1 shows $\sigma_{x}^{2}$ and $\sigma_{y}^{2}$ as functions of the forcing amplitude $z_{0}$ [see Part I, Eq. (4a)]. The $\sigma_{y}^{2}$ behavior is easily understood: the meridional dispersion increases with the perturbation amplitude. This increase in $\sigma_{y}^{2}$ simply reflects the expansion in the width of the surf zone, which is very clearly seen in the corresponding tracer fields (cf. Part I, Fig. 4). It is analogous to the behavior of kinematic chaotic advection models: $\sigma_{y}^{2}$ increases with the perturbation amplitude $\varepsilon$ due to the expansion in the width of the chaotic region (cf. Ngan and Shepherd 1997a, Fig. 13). There is, however, a fundamental difference between the systems: in kinematic chaotic advection models, one has invariant tori that are fixed in place on a Poincaré section; in the shallow-water system unbroken potential vorticity (PV) contours are time dependent and deform as the critical layer evolves. Nevertheless,

\footnotetext{
${ }^{1}$ Note that in computing $\sigma_{x}^{2}$ the periodic boundary conditions are ignored. This is a standard technique in dispersion studies (see, e.g., Mezić and Wiggins 1994).
}

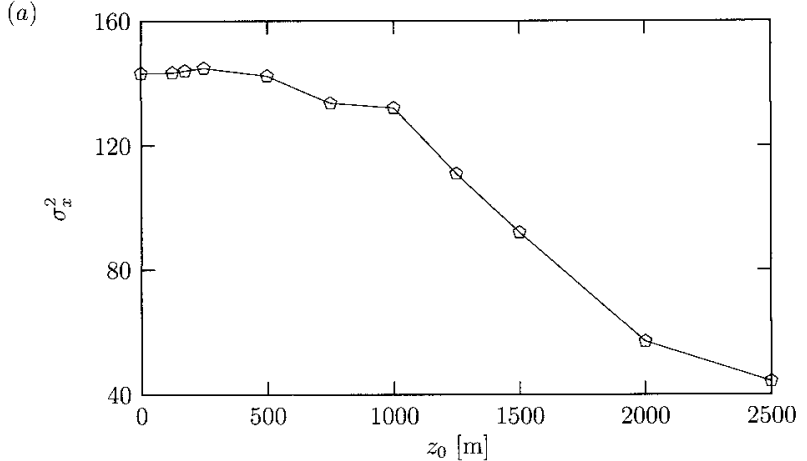

(b)

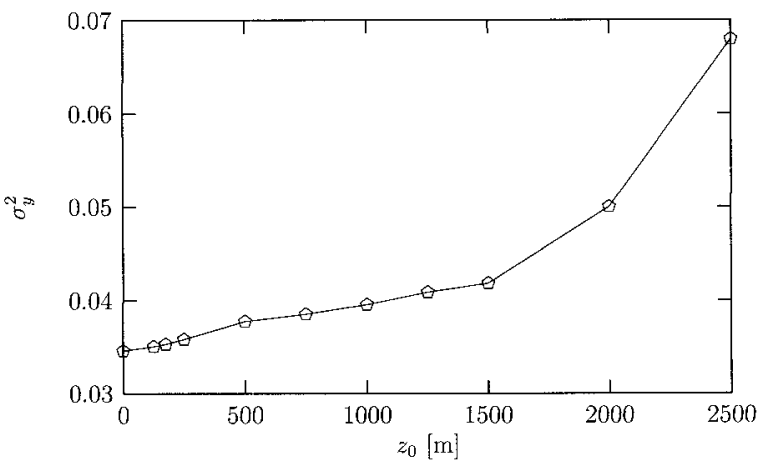

FIG. 1. Dispersion statistics at 50 days as a function of $z_{0}$ : (a) $\sigma_{x}^{2}$; (b) $\sigma_{y}^{2}$.

this does demonstrate that there is some justification in thinking of the surf zone as a "chaotic region" and the rest of the flow as a "regular region."

The $\sigma_{x}^{2}$ behavior is a little more complicated: the dependence on $z_{0}$ is not determined solely by the expansion in the width of the surf zone; the orientation of the initial particle distribution relative to the surf zone is also important. For small $z_{0}$, some of the particles lie in a region of quasi-zonal, nonbreaking streamlines, the rest in a region of overturning, breaking streamlines (i.e., the surf zone); for large $z_{0}$, however, all the particles are contained in the surf zone, the initial distribution being fixed. This is important because the zonal dispersion is maximized when the particles lie in regions with different Lagrangian-mean zonal velocities (see section $2 b$ ): in the weak-forcing case particles drift at different average rates in different regions and $\sigma_{x}^{2}$ is large; in the strong-forcing case, the particles lie in the same region and therefore drift at the same average rate. There is once more similar behavior for deterministic chaotic advection models (cf. Ngan 1997).

Plots of $\sigma_{x}^{2}$ and $\sigma_{y}^{2}$ versus time (Fig. 2) show that the temporal development of the dispersion statistics is also governed by the underlying phase-space structure. For $\sigma_{x}^{2}$, it is evident that the decrease in $\sigma_{x}^{2}(t=50$ days $)$ with $z_{0}$ (as seen in Fig. 1) is a manifestation of the corresponding decrease in the dispersion exponent $\gamma$, which is defined by $\sigma_{x}^{2} \sim t^{\gamma}$. In recent years there has been much interest in computing $\gamma$ for a number of 
(a)

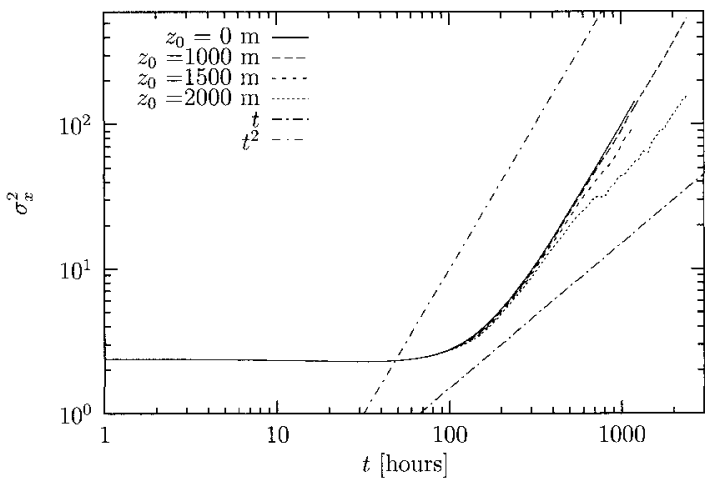

(b)

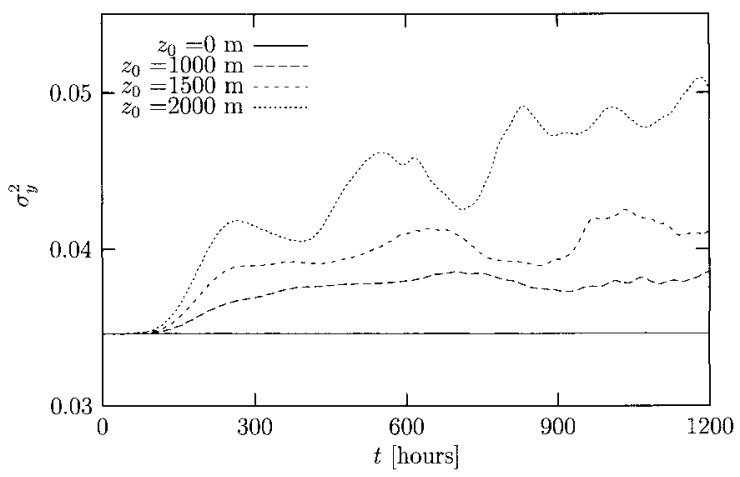

FIG. 2. Dispersion statistics as a function of time for $z_{0}=0,1000$, 1500 , and $2000 \mathrm{~m}$ : (a) $\sigma_{x}^{2}$; (b) $\sigma_{y}^{2}$. Note that in (a) logarithmic axes are used, and the results for $z_{0}=1000$ and $2000 \mathrm{~m}$ extend to 100 days.

different systems (see Mezić and Wiggins 1994 and references therein). For $\sigma_{y}^{2}$, by contrast, one sees the initial growth and long-time saturation of the meridional dispersion, the time of saturation being greater for larger $z_{0}$.

It is interesting to examine how this picture changes with the external parameters. In Part I it was argued that the formation of cat's eyes in the surf zone is robust to changes in the basic-state shear and the deformation radius, as well as to the addition of transient forcing.

For transient forcing alone the results are qualitatively similar: $\sigma_{x}^{2}$ scales very nearly as $t^{2} ; \sigma_{y}^{2}$ increases with time and then saturates. The differences are greater for $\sigma_{y}^{2}$ because $\sigma_{x}^{2}$ is evidently insensitive to the small-scale structure of the velocity field (see section $2 b$ ). There are similar results when the deformation radius and the basic-state shear are changed: the dispersion statistics are robust because the large-scale velocity field is robust.

For small forcing amplitudes stochastic forcing has a similar effect. This may be seen in Fig. 3, which shows $\sigma_{y}^{2}$ at 50 days as a function of the stochastic forcing amplitude in the velocity components, $\delta_{v} .{ }^{2}$ Note that

\footnotetext{
${ }^{2}$ More precisely $\delta_{v}$ is defined by the equation $v \rightarrow v\left(1+\delta_{v} \eta\right)$, where $v$ is a velocity component and $\eta$ is a white-noise variable, in both space and time, of amplitude unity. See Part I, Eq. (5).
}

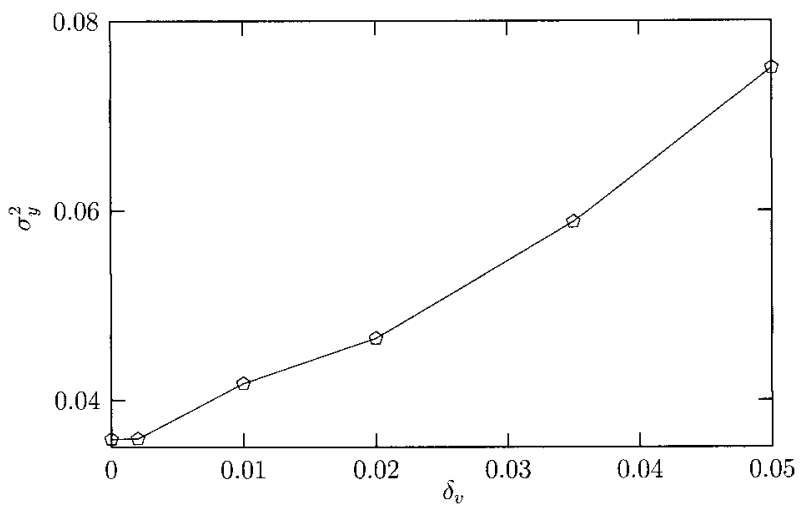

FIG. 3. $\sigma_{y}^{2}(t=50 \mathrm{~d})$ vs $\delta_{v} \cdot z_{0}=250 \mathrm{~m}$.

$\sigma_{y}^{2}$ increases steadily with $\delta_{v}$; plots of $\sigma_{y}^{2}$ against time (not shown) confirm that in this range of parameters, $\sigma_{y}^{2}$ once more increases and levels off with time, albeit slowly.

This behavior is analogous to that of the stochastic two-wave model of Ngan and Shepherd (1997a). In that model stochasticity in the phase speed of the transient perturbation causes particle trajectories to be smeared out, but as the large-scale structure of the velocity field is unaffected, the behavior of $\sigma_{y}^{2}$, a coarse-grained diagnostic, is fairly predictable: $\sigma_{y}^{2}$ generally increases with stochasticity. There is a similar situation here for weak stochastic forcing: after all, the cat's eye structure in the surf zone does persist. The situation for strong forcing is different, however, since in that case the cat's eye structure is essentially destroyed; the dispersion behavior changes along with the large-scale velocity field; $\sigma_{y}^{2}$ increases rapidly. We return to this point in section 4 , where Liapunov exponents are computed.

\section{b. Anomalous diffusion}

In the preceding section it was shown that for the zonal dispersion $\gamma \approx 2$. This result is perhaps surprising because one might expect $\gamma=1$ (i.e., normal diffusion) in a chaotic region; we therefore consider the scaling of $\sigma_{x}^{2}$ in detail.

There is a simple physical explanation for the occurrence of $t^{2}$ or $t^{1}$ dispersion. The $t^{1}$ dispersion occurs when the Lagrangian-mean zonal velocities of the particles approach a constant for $t \rightarrow \infty$ (assuming that the zonal velocities are sampled randomly, the variance of the ensemble positions then grows like that of a onedimensional random walk); $t^{2}$ dispersion, sometimes referred to as ballistic motion, occurs when particles in regions with different Lagrangian-mean zonal velocities separate from one another linearly in time. In other words, $t^{1}$ dispersion is expected for times large in comparison with the Lagrangian autocorrelation time; if the latter is infinite, $t^{2}$ dispersion results. The nonergodicity condition of Mezić and Wiggins (1994) formalizes this physical argument. 
The type of dispersion observed thus reflects the structure of the velocity field. From the argument given above, $t^{2}$ and $t^{1}$ dispersion are limiting cases as $t \rightarrow \infty$ for (quasiperiodic) "regular" and "irregular" velocity fields, respectively. ${ }^{3}$ At finite times, dispersion exponents between 1 and 2 may be possible. This is referred to as anomalous diffusion ${ }^{4}$ and is frequently associated, in non-Hamiltonian systems, with stochasticity in the velocity field (Crisanti and Vulpiani 1993), or, in Hamiltonian systems, with Lévy flights, wherein particle displacements alternate between "trapping" and "flight" episodes (Shlesinger et al. 1993).

These considerations are of course theoretical only. In practice, it is often difficult to observe $\gamma<2$, let alone verify claims of anomalous diffusion, because the time series are relatively short. Assuming a characteristic velocity of $30 \mathrm{~m} \mathrm{~s}^{-1}$ and a characteristic length scale of $a \pi / 3$ ( $a$ is the radius of the earth), the characteristic advective timescale of the large-scale flow is of the order of 3 days, implying that a 50-day run corresponds to roughly 16 advective periods. This means that 50-day integrations are too short for there to be diffusive-like motion in the zonal direction. After 50 days the particles are dispersed over the surf zone along a number of convoluted but nonetheless well-defined filaments (Part I, Fig. 2c); consequently the particles do not sample the zonal velocities randomly and the Lagrangian-mean zonal velocities cannot approach a constant. Even for a 100-day integration (Fig. 2a), the integrations are still too short for the zonal motion to be diffusive-like, although $\gamma \sim 1.4$ for $z_{0}=2000 \mathrm{~m}$ and $t>900 \mathrm{~h}$. On the other hand, for $z_{0}=1000 \mathrm{~m}, \gamma=$ 2 still (however, $\gamma<2$ can be obtained if the initial distribution is sufficiently narrow; not shown).

While this behavior is suggestive of anomalous diffusion, it may be that $\gamma \rightarrow 1$ for larger $t$. In deterministic Hamiltonian systems anomalous diffusion is a finitetime effect. The occurrence of anomalous diffusion would be interesting because it might be indicative of a qualitative change in the spatial structure of the velocity field. However, $\sigma_{x}^{2}$ is a rather coarse diagnostic: $\sigma_{x}^{2} \sim t^{2}$ as $t \rightarrow \infty$ even if only a small fraction of the particles is located outside the breaking region, as discussed above.

For cases with explicit stochastic forcing, one might expect anomalous diffusion to occur: the cat's eye structure is destroved by strong stochasticity and there is much spatial irregularity in the velocity field (see Part $\mathrm{I}$, section 3e). At least for a 50-day integration, however, this does not appear to be the case for the standard

\footnotetext{
${ }^{3}$ We emphasize that it is not necessary for the entire velocity field to be regular for there to be $t^{2}$ dispersion; only part of the tracer field need be contained in a regular region.

${ }^{4}$ The term anomalous diffusion is used even for cases where there is no explicit diffusion of the tracer. While "anomalous dispersion" may be a more accurate description, this usage is standard.
}

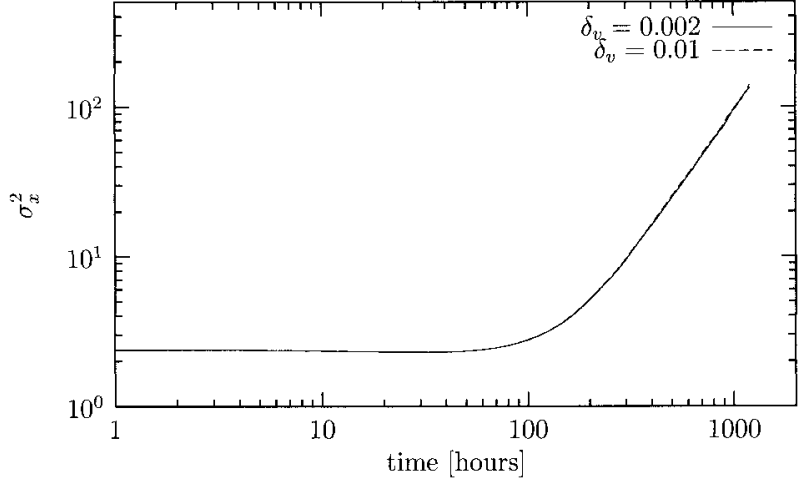

FIG. 4. $\sigma_{x}^{2}$ vs time for $\delta_{v}=0.002$ and $\delta_{v}=0.01 . z_{0}=1000 \mathrm{~m}$.

choice of parameters; $\gamma=2$ yet again (Fig. 4). It is plausible that $\gamma=2$ is a robust result for strong stochastic forcing. For the stochastic two-wave model $\gamma=$ 2 for large $t$ because while the zonal shear is unaffected by stochasticity, particles tend to drift away from one another in the meridional direction, making $t^{2}$ dispersion inevitable. Here the structure of the zonal jet is basically unaffected by stochasticity, and the same kind of mechanism should be operative.

We note that there is little evidence for the existence of Lévy flights in the shallow-water system. With or without stochasticity, there is not a clear-cut distinction between trapping and flight episodes; instead, particle displacements vary continuously with time.

\section{Transport fluxes}

Dispersion statistics are a useful diagnostic for transport but they are not the only one. For Hamiltonian dynamical systems, analytical theory ("lobe dynamics") for transport across the perturbed separatrix has been developed (e.g., Wiggins 1992). This provides a means of relating the phase-space structure to the measured transport. In the stratospheric context, much attention has been devoted to measures of transport across the edge of the polar vortex; an entirely satisfactory one has yet to be devised (Sobel et al. 1997). Nevertheless it is useful to compute brute-force transport fluxes across some arbitrarily chosen boundaries: our interest is not in transport per se but rather in its relation to the largescale velocity field, that is, as a manifestation of chaotic advection.

The initial particle distribution is chosen according to the appearance of the tracer field at 50 days, specifically the location of the cat's eyes. As in Ngan and Shepherd (1997a) the number of particles crossing the northern and southern boundary of the initial distribution is counted; fluxes are obtained by normalizing this number with respect to the number of particles in the initial distribution. The qualitative results depend on neither the precise location of the boundaries nor the number of particles. 


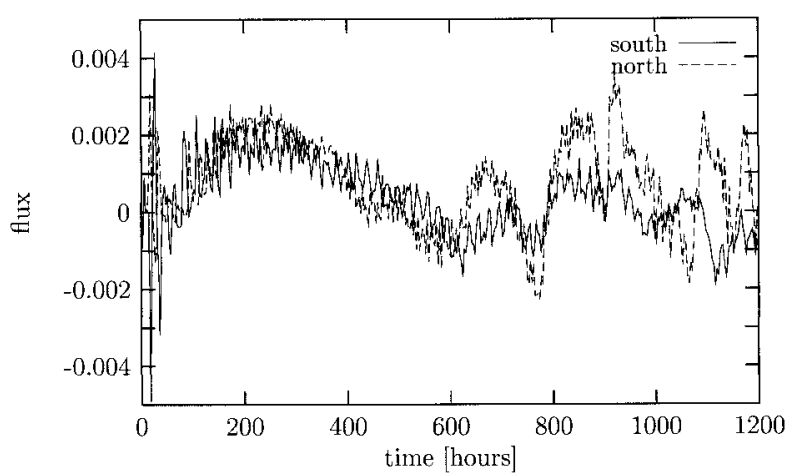

FIG. 5. Transport fluxes for $z_{0}=1000 \mathrm{~m}$ and an initial particle distribution between $20^{\circ}$ and $35^{\circ} \mathrm{N}$. Positive fluxes are northward at the northern boundary and southward at the southern boundary.

Figure 5 shows the transport fluxes for $z_{0}=1000 \mathrm{~m}$ and an initial particle distribution between $20^{\circ}$ and $35^{\circ} \mathrm{N}$. In contrast with the fluxes from the deterministic twowave model (obtained from lobe dynamics or bruteforce computation; see Ngan and Shepherd 1997a), there is no sign of power-law scaling: instead the flux across either boundary evolves on a slow timescale, of the order of 20 days, with inertial oscillations superimposed on top. Similar results have been obtained with other initial distributions and with longer, 100-day, runs.

This behavior is a consequence of the time dependent nature of the critical layer. To see this it is necessary to recall why there is power-law decay of transport fluxes in dynamical systems. Temporal power laws are associated with self-similar phenomena, that is, processes without a characteristic scale. In systems with periodic boundary conditions, the chaotic motion around the separatrix, which does not have a characteristic timescale, presumably leads to power-law decay (cf. Ngan and Shepherd 1997a); for unbounded systems, by contrast, exponential decay is possible (cf. Rom-Kedar et al. 1990). The shallow-water system falls in between these two extremes (power spectra of velocity time series show broad peaks; Part I, section 4a) and this likely explains its more complicated time dependence. Similar kinds of time dependence have been obtained in previous studies (cf. Sobel et al. 1997; Polvani et al. 1995): the transport fluxes are time dependent and show "bursts" of increased transport.

The scaling of the transport fluxes in systems with complicated time dependence has yet to be studied systematically. This diagnostic illustrates how chaotic advection in kinematic and in dynamical models may differ. In quasiperiodic studies (e.g., Duan and Wiggins 1996), the time evolution of an averaged quasiperiodic Melnikov function is generally weak. ${ }^{5}$ This is very dif-

\footnotetext{
${ }^{5}$ It should be pointed out, however, that the Melnikov function describes the total phase space transport rather than the transport of a particular tracer (cf. Ngan and Shepherd 1997a). The Melnikov function provides an estimate of the separatrix splitting and thus the phase-space transport across the separatrix.
}

ferent from the shallow-water system, where the spatial structure of the cat's eye structure is not fixed in time (see Part I, Fig. 2).

\section{Liapunov exponents}

We now turn to the mixing diagnostics. Mixing in chaotic systems is often characterized using finite-time Liapunov exponents (hereafter abbreviated to Liapunov exponents for brevity), which roughly speaking measure the exponential divergence of two nearby trajectories, that is, the stretching characteristics of the flow in question.

In a chaotic region there is a distribution of Liapunov exponents at any finite time. This distribution may be represented using a probability density function (PDF), $P(\lambda){ }^{6}$ Although the direct implications of $P(\lambda)$ for the mixing of a tracer field are tenuous, it is, nevertheless, useful in understanding the nature of chaotic advection in the shallow-water system and the middle-atmosphere model. There has recently been much study of the Liapunov exponents of chaotic systems, both deterministic and stochastic (e.g., Városi et al. 1991), as well as of random-strain theory (e.g., Chertkov et al. 1995). By examining the form and evolution of the PDFs, as well as their first moment (i.e., the mean Liapunov exponent), both differences and similarities between chaotic advection in a realistic vorticity-conserving fluid and in a kinematic model can be established. In particular the relationship of stratospheric mixing and transport to chaotic advection and random-strain theory, which was partially addressed in Part I, is further clarified.

The Liapunov exponents are computed using the socalled pull-back method (see Lichtenberg and Lieberman 1992), wherein an initial perturbation $\delta x(0)$ is chosen at random and allowed to evolve under the linearized dynamics. This shadow trajectory is displaced by a maximum distance (in radians) of $\left(\delta_{\lambda}, \delta_{\phi}\right)=(2 \mathrm{E}-5,1 \mathrm{E}-5)$ and is renormalized every 37.5 minutes. ${ }^{7}$ The results are not sensitive to the values of these parameters. Comparable results have been obtained using a matrix-based method (von Bremen et al. 1997; Geist et al. 1990), wherein the Liapunov exponents are obtained as eigenvalues of what is essentially an averaged displacement matrix, as well as with the full nonlinear flow.

The PDFs are constructed by binning the Liapunov exponents between $\lambda=-1$ and $\lambda=1$ day $^{-1}$ into 400 equally spaced bins and normalizing. Unless stated otherwise the initial distribution is the same as that in section 2.

\footnotetext{
${ }^{6}$ Hereinafter $\lambda$ refers to the Liapunov exponent, not the longitude.

${ }^{7}$ The offline winds are sampled every $3 \mathrm{~h}$ and the time step of the particle advection scheme is $3.75 \mathrm{~min}$.
} 


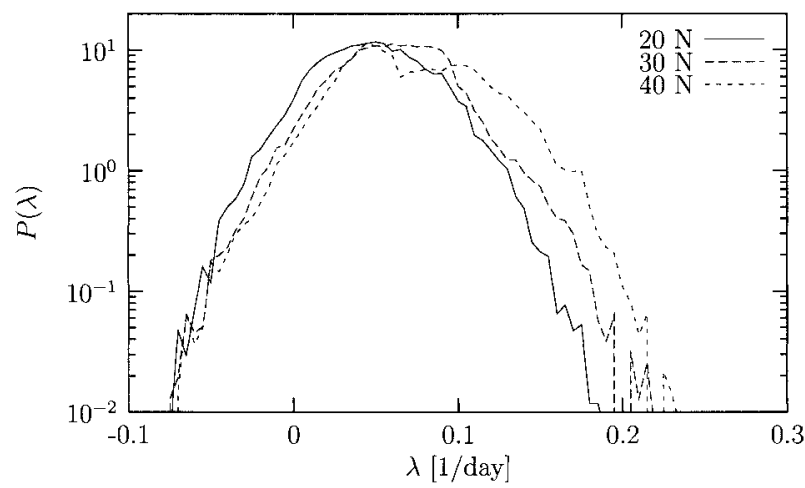

FIG. 6. $P(\lambda)$ at $t=50$ days for $\phi_{0}=20^{\circ}, 30^{\circ}$, and $40^{\circ} \mathrm{N} . z_{0}=$ $1000 \mathrm{~m}$.

\section{a. Comparison with contour-lengthening exponents}

Before proceeding with a detailed analysis of the Liapunov exponents, it is useful to see how they compare to the contour-lengthening exponent, a diagnostic commonly used to characterize stratospheric mixing (e.g., Waugh and Plumb 1994; Polvani et al. 1995).

The contour-lengthening exponent is a useful quantity to examine because it provides information about the development of small-scale tracer structure. However, it does have two notable limitations. The first is that it is a coarse-grained quantity: the contour-lengthening rate is often supposed to be a Liapunov exponent (e.g., Schoeberl and Newman 1995), but this is not strictly correct. The Liapunov exponent, as conventionally defined, is a fine-grained diagnostic, whereas the contourlengthening rate is obviously related to the distribution of Liapunov exponents along the contour. Second, the contour-lengthening rate is obtained as a function only of the initial contour location, $\phi_{0}$. While this enables the edges of the surf zone to be demarcated, information about the spatial distribution of mixing is lost: the Liapunov exponents along the contours are not, in general, identical.

These points are illustrated by comparing the contourlengthening exponents of the shallow-water system to the corresponding PDFs. For brevity we only sketch the procedure used to obtain the contour-lengthening exponents: the initial location of a (potential vorticity) contour is specified by $\phi_{0}$; the evolution of the contour is followed using the contour-advection scheme of Schoeberl and Newman (1995) with a cut-off threshold of $100 \mathrm{~km}$; the contour length is obtained by summing over the particle separations. From a least squares fit the contour-lengthening rates for 50-day integration with $z_{0}=1000 \mathrm{~m}$ are $0.11 \pm 0.02,0.13 \pm 0.02$, and $0.15 \pm 0.03 \mathrm{day}^{-1}$ for $\phi_{0}=20^{\circ}, 30^{\circ}$, and $40^{\circ} \mathrm{N}$, respectively (the errors are estimated from minimum and maximum slopes). Typical values for the lower stratosphere are of the order of 0.2 day $^{-1}$ (e.g., Schoeberl and Newman 1995).

Figure 6 shows $P(\lambda)$ at $t=50$ days for $\phi_{0}=20^{\circ}$, $30^{\circ}$, and $40^{\circ} \mathrm{N}$. While it is difficult to compare the values of the Liapunov exponents to the contour-lengthening rates - the former being determined from a 50-day integration and the latter from a least squares fit at large times, the range of times considered being somewhat arbitrary - the contour-lengthening rates do appear to be greater. This is probably because the stretching of a finite fluid element is in general greater than the stretching of an infinitesimal fluid element (e.g., Tabor and Klapper 1994): if there is nonuniform stretching then the lengthening of a contour may be determined by a small number of rapidly stretching elements.

This may be seen in the following way. The ensembleaveraged Liapunov exponent at time $t$ is given by

$$
\langle\lambda\rangle=\int P(\lambda, t) d \lambda,
$$

while the contour-lengthening rate at time $t$ is

$$
\lambda_{c} \sim \frac{1}{t} \ln \left\{\int \exp (\lambda t) P(\lambda, t) d \lambda\right\} .
$$

Because of the exponential factor, larger values of $\lambda$ make a larger contribution to the integral. This can be made concrete by taking the infinite-time limit of random-strain theory (see section 4b). In this limit $P(\lambda, t)$ approaches a Gaussian, that is, $P(\lambda) \sim \exp \left(-(\lambda-\langle\lambda\rangle)^{2} /\right.$ $\left.\sigma_{\lambda}\right), \sigma_{\lambda}$ being the standard deviation of the distribution. If $\sigma_{\lambda}$ is small, it is then easy to show that the dominant contribution to the integral is given by a value of $\lambda$ displaced from $\langle\lambda\rangle$ by a positive distance.

Another point is that $P(\lambda)$ contains more information than the contour-lengthening rate, which is just a number. Here $P(\lambda)$ provides information about the homogeneity of the mixing: broad distributions imply inhomogeneous mixing. While this is statistical information only, it is nonetheless more useful than a distribution of contour-lengthening rates with respect to $\phi_{0}$. The latter may be somewhat misleading because it is a distribution in the space of initial contour locations, which can be very different from the final tracer field.

These points are elementary and have been recognized, implicitly, by several previous authors (e.g., Haynes and Anglade 1997). The purpose of the preceding calculations is mostly pedagogical: they provide explicit evidence that the contour-lengthening rate is an inherently coarse-grained diagnostic and they help motivate the analysis in the subsequent sections.

\section{b. Probability distribution}

Figure 7 shows $P(\lambda)$ at 50 days for $z_{0}=1000,2000$, and $3000 \mathrm{~m}$. In all cases one sees a rather broad PDF that is skewed toward positive $\lambda$. As $z_{0}$ is increased, the mean of the distribution shifts slightly to larger $\lambda$ (see section $4 \mathrm{c}$ ), and the large- $\lambda$ tails become more pronounced. 


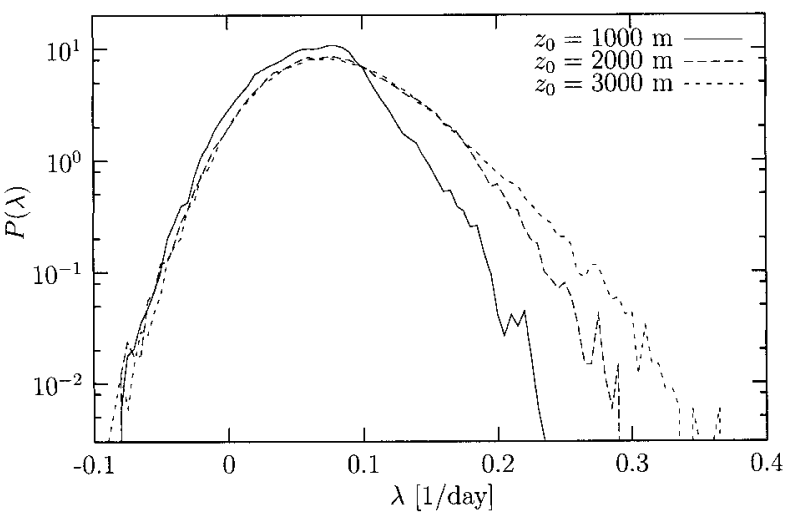

FIG. 7. $P(\lambda)$ at 50 days for $z_{0}=1000,2000$, and $3000 \mathrm{~m}$.

A complementary picture is obtained from the time evolution for a single value of $z_{0}$ (Fig. 8). The large- $\lambda$ tail is much diminished at 100 days and the approach toward Gaussianity is clear.

This behavior may be understood by appealing to some theoretical results. It has been shown that for a two-dimensional, incompressible flow with strain components varying randomly in time $P(\lambda)$ takes the following form (Chertkov et al. 1995):

$$
P_{\mathrm{rs}}(\lambda) \sim \exp \left[-\alpha \frac{(\lambda-\bar{\lambda})^{2} \ln \left(L / r_{\min }\right)}{2 \Delta_{\lambda} \lambda}\right],
$$

where $\alpha$ is an inverse time, $L$ is a correlation length scale of the flow, $r_{\min }$ is a minimum tracer length scale, and $\bar{\lambda}$ and $\Delta_{\lambda}$ are the mean and variance of the distribution for $t \rightarrow \infty$. At large times $P_{\mathrm{rs}}(\lambda)$ approaches a Gaussian since $L / r_{\min }$ is large $\left[\bar{\lambda} t \sim \ln \left(L / r_{\min }\right)\right]$ and $\Delta_{\lambda}$ is effectively small; at intermediate times $P_{\mathrm{rs}}(\lambda)$ is skewed toward increasing $\lambda$.

As predicted by (4), the PDFs in Figs. 7 and 8 exhibit positive skewness at finite times and collapse toward Gaussianity as time increases. Quantitative comparison is difficult-(4) is basically a scaling expression and has little predictive value - but this approximate agreement is interesting because random-strain theory is a rather severe idealization. It would appear that violation of a number of its underlying assumptions does not, at least on the timescales considered here, yield significant deviations. On longer timescales effects such as the nonvanishing divergence field and, more importantly, the presence of transport barriers at the edges of the surf zone could be important - the collapse to Gaussianity might be incomplete, for example-but they are, evidently, unimportant on timescales relevant to stratospheric mixing and transport. What is important is that random-strain theory does seem to apply reasonably well, in accord with the ideas of Juckes and McIntyre (1987) and Haynes and Anglade (1997). For example, spatial correlations in the velocity field, which are excluded in random-strain theory, could be an issue on short timescales, but the Lagrangian correlation time

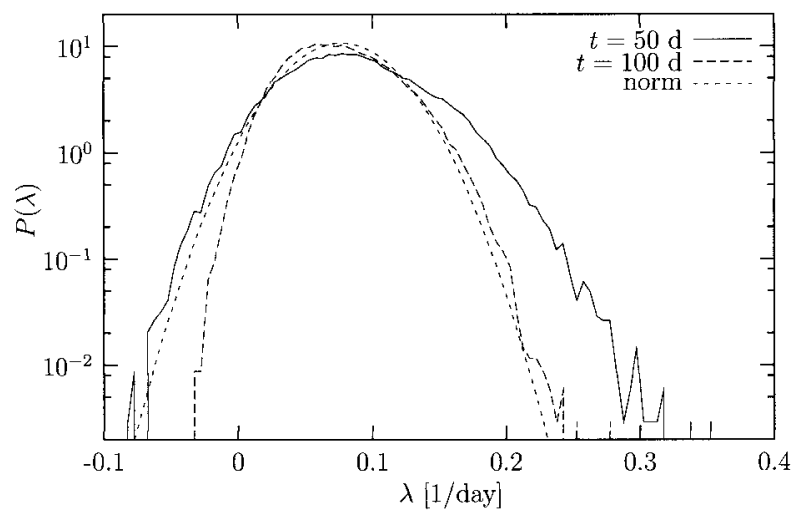

FIG. 8. $P(\lambda)$ for $z_{0}=2000 \mathrm{~m}$ at 50 and 100 days. A normal distribution is shown for comparison.

(see Part I, section 4) is probably short enough for this to be a minor effect (see section 4c below as well), though deviations from Gaussianity cannot be ignored altogether. Likewise the absence of a single precisely defined correlation time- the $u_{y}$ derivative decorrelates more slowly than the other components (Part I, Fig. 13) - has minimal effect at large times.

Another way of seeing this is that the emergence of a (near) Gaussian PDF is a generic phenomenon that does not require random strain per se. Basically, a Gaussian PDF is the outcome of a central-limit theorem and, for practical purposes, it is immaterial how exactly the requisite randomness is introduced; one sees similar behavior for stochastically forced chaotic systems, for example (Városi et al. 1991; Ngan and Shepherd 1997b). ${ }^{8}$ While the shallow-water system is certainly very different from these idealized models, it nonetheless exhibits Lagrangian random strain (and chaotic advection), and its statistical behavior reflects this correspondence.

We now consider the robustness of these results. For stationary and transient forcing the PDFs compare very well: the small differences that appear in the corresponding tracer fields do not reflect qualitative changes in the mixing characteristics. The results are much the same when the deformation radius is varied; the cat's eye structure persists and similar PDFs obtain.

There is, however, a quantitative change in the PDFs when the basic-state shear is altered (Fig. 9). While the qualitative behavior is robust, there is more mixing for doubled shear (the peak of the distribution shifts to larger $\lambda$ ) and less mixing for halved shear (the peak shifts to smaller $\lambda$ ); these shifts are of roughly the same magnitude and are consistent with the corresponding tracer fields (see Part I, Fig. 5). The PDF for the halved case is significantly narrower than the others; this is reminiscent of Fig. 7, where the width of the PDF expands

\footnotetext{
${ }^{8}$ Without stochasticity, however, the PDFs show negative skewness due to the presence of KAM tori (cf. Horita et al. 1990).
} 


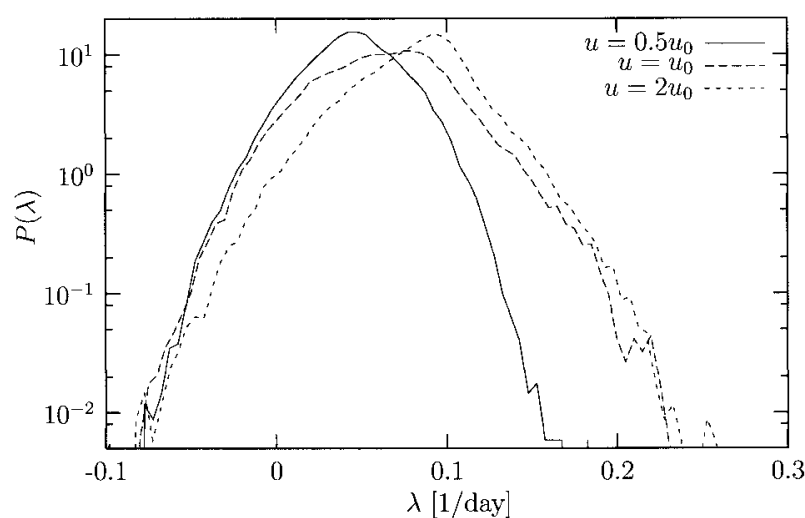

FIG. 9. $P(\lambda)$ at 50 days for basic states with doubled and halved shear. $u_{0}$ refers to the initial velocity of the zonal jet [see Part I, Eq. (1) $] ; z_{0}=1000 \mathrm{~m}$.

with $z_{0}$. With stronger shear (or forcing) a greater range of stretchings is possible, meaning that, everything else being the same, convergence toward a limiting value of $\lambda$ is slowed, and longer tails result.

It is interesting to examine the PDFs for a case in which a well-defined cat's eye structure does not exist. In Fig. 10, for which $\delta_{v}=0.05$, the cat's eye structure is wiped out and the PDFs are close to Gaussian after only 25 days. Although it is not proper to speak of a large-scale velocity field here (see Part I, Fig. 12), a mechanism similar to that embodied by (4) would appear to be operative: the collapse toward Gaussianity is almost complete because the correlation time of the velocity derivatives, Lagrangian or Eulerian, is short (effectively, the integrations are long). This behavior is in marked contrast to that of the PDFs studied earlier (e.g., Fig. 8), which show a more complicated time evolution. Physically, this time evolution of the PDFs simply reflects the fact that the velocity field is inhomogeneous: the evolution is speeded up when stochasticity is added because inhomogeneities are reduced. This trivial observation explains why differences between the present trajectory-based method used to compute the Liapunov exponents and a matrix-based method (not shown) are smaller for the stochastic runs: when the flow is stochastic the instantaneous eigenvalues provide a better approximation to the net stretching experienced along a trajectory.

The foregoing suggests that strong stochasticity does not alter the PDFs qualitatively: its main effect is to accelerate the approach toward Gaussianity. However, there is an important quantitative effect, namely, that the computed value of the Liapunov exponents is altered. The Liapunov exponents increase with $\delta_{v}$ and, perhaps even more significantly, this increase is dependent on spatial resolution: the probability distribution is sensitive to small-scale fluctuations. We illustrate this in Fig. 11. In the absence of stochasticity the PDFs are robust: high-resolution velocity fields yield nearly identical PDFs, as required (Fig. 11a). When stochasticity

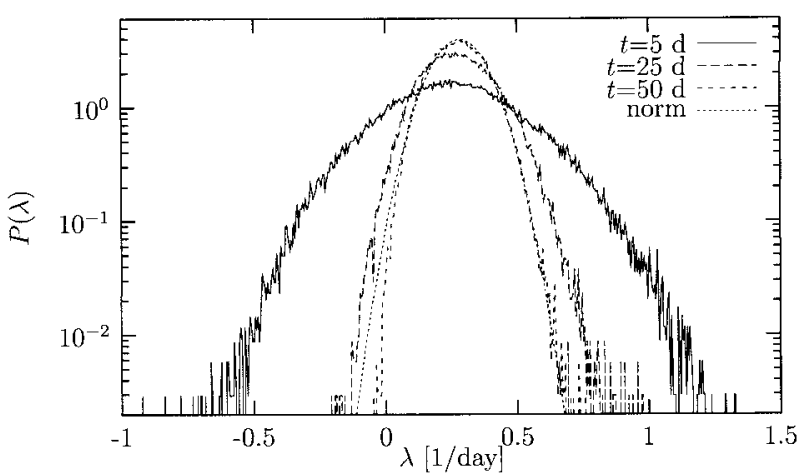

FIG. 10. Time evolution of $P(\lambda)$ for strong stochastic forcing $\left(z_{0}\right.$ $\left.=250 \mathrm{~m}, \delta_{v}=0.05\right)$. The corresponding normal distribution at 50 days is also shown.

is added, however, the results are sensitive to spatial resolution (Fig. 11b), though there is good agreement between realizations. In this case, which is clearly untypical of the wintertime lower stratosphere, the cat's eye structure is destroyed and it is no longer true that small-scale tracer features are generated by a large-scale velocity field. These results imply that, from a practical point of view, off line tracer calculations cannot be reliably carried out when there is strong small-scale variability; from a theoretical perspective they demonstrate how chaotic advection can be suppressed.
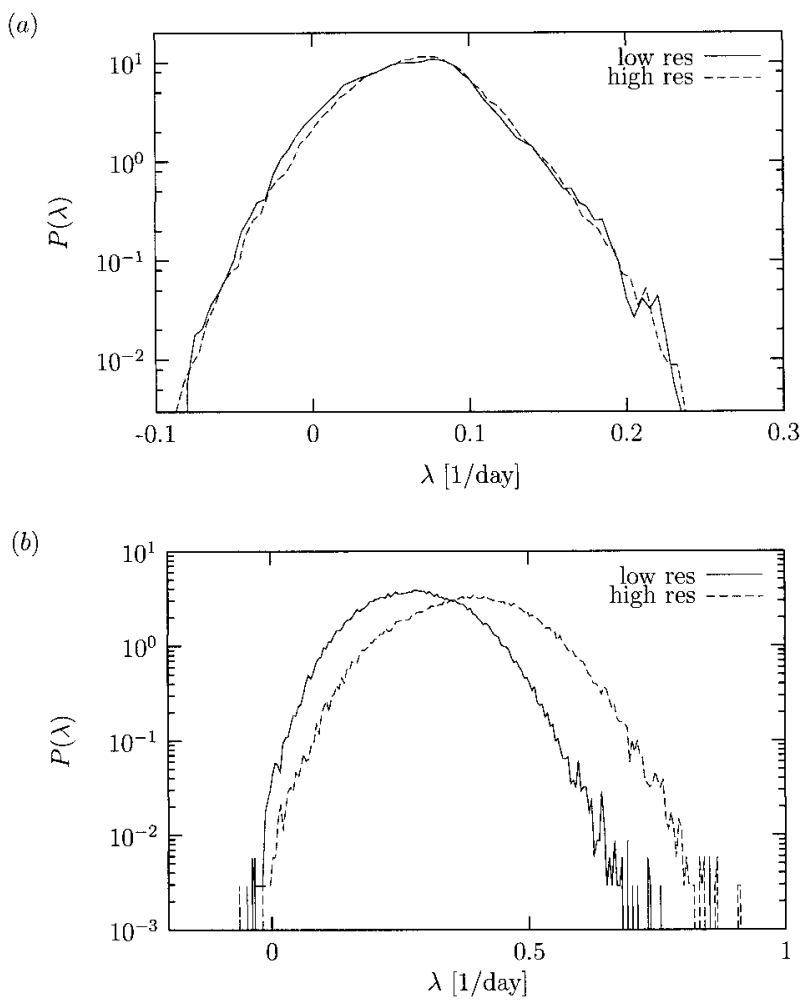

FIG. 11. Effect of spatial resolution on $P(\lambda)$ at 50 days. (a) Stationary forcing, $z_{0}=1000 \mathrm{~m}$; (b) strong stochastic forcing, $z_{0}=250$ $\mathrm{m}$ and $\delta_{v}=0.05$. 


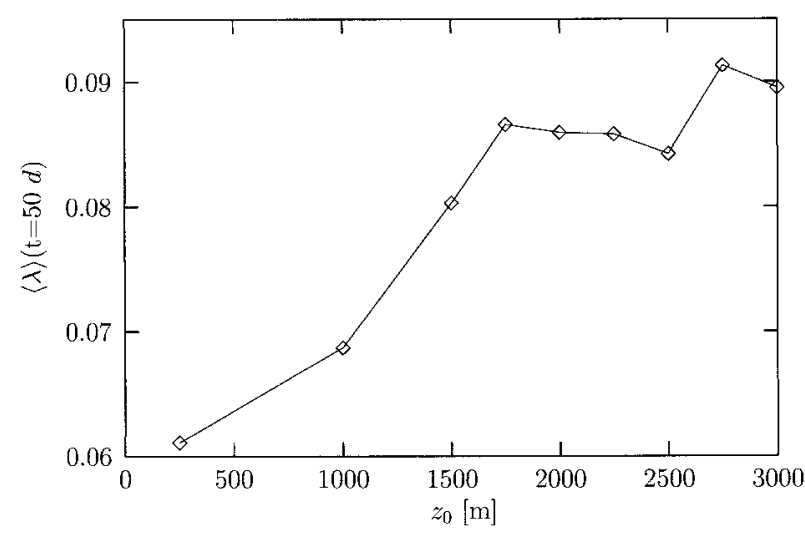

FIG. 12. $\langle\lambda\rangle(t=50 \mathrm{~d})$ vs $z_{0}$.

\section{c. Scaling of the ensemble average}

The scaling of the ensemble-averaged Liapunov exponent $\langle\lambda\rangle$ with respect to $z_{0}$ and $\delta_{v}$ is now considered. This allows us to quantify some of the trends touched upon above, for instance, the shift of $P(\lambda)$ with $z_{0}$ (cf. Fig. 7). Moreover, it enables the applicability of randomstrain arguments to be further examined.

In Fig. 12 we plot $\langle\lambda\rangle$ as a function of $z_{0}$. There is a general increasing trend, though the increase is relatively small considering the range of $z_{0}$. Specifically, $\langle\lambda\rangle$ initially increases with $\lambda$, levels off for $1750<z_{0}$ $<2500 \mathrm{~m}$, and increases slightly once more for larger $z_{0}$.

This behavior of $\langle\lambda\rangle$ is determined by several competing influences. In random-strain theory, $\langle\lambda\rangle$ is determined by the mean strain and vorticity $(S$ and $\Omega$, respectively), as well as their associated correlation time $\tau_{\mathrm{rs}}$. Chertkov et al. (1995) show that for $t \rightarrow \infty$,

$$
\langle\lambda\rangle\left(\tau_{\mathrm{rs}}\right)=S \tanh \frac{S \tau_{\mathrm{rs}}}{1+\Omega \tau_{\mathrm{rs}}},
$$

by interpolating between the limits of small and large $\tau_{\mathrm{rs}}$. As with (4), this equation cannot be applied directly (the parameters are computable, but difficult to obtain precisely). Rather it provides some useful guidance. Since the Lagrangian correlation time is basically independent of $z_{0}$ (Part I, Fig. 14), it follows that in this case $\langle\lambda\rangle$ is determined mostly by $S$ and $\Omega$.

The strain (and vorticity) may increase with $z_{0}$ due to generation of stronger velocity gradients. Another factor, which may be more important here, is that the width of the surf zone expands. Since the strongest mean shear occurs to the north of the zero-wind line (Part I, Fig. 1), the increase in $\langle\lambda\rangle$ from $z_{0}=250$ to $1000 \mathrm{~m}$ is plausibly connected to the large expansion in the size of the surf zone (Part I, Figs. 2 and 4) and the concomitant sampling of stronger velocity gradients. The relatively small variations in $\langle\lambda\rangle$ between 1750 and 2500 $\mathrm{m}$, by contrast, reflect the fact that the width of the surf zone is approximately constant. For large $z_{0}$, the increase in the magnitude of the velocity derivatives could

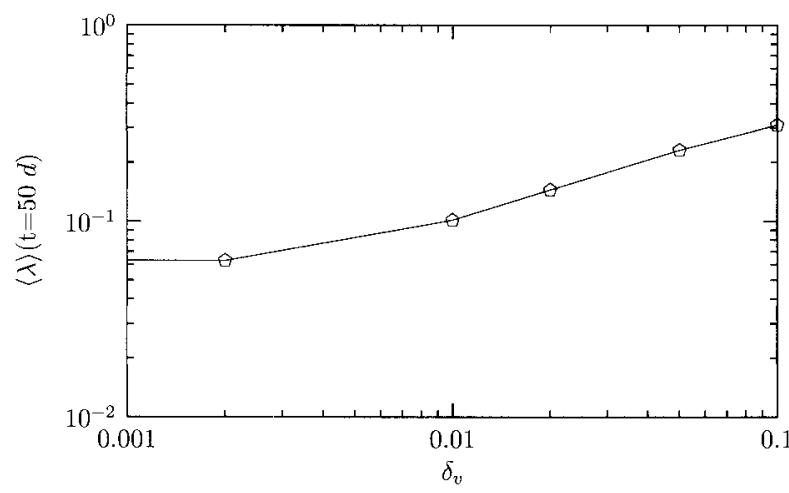

FIG. 13. $\langle\lambda\rangle(t=50 \mathrm{~d})$ vs $\delta_{v}$ on $\log -\log$ axes. $z_{0}=250 \mathrm{~m}$.

be important since the vortex splits and large-scale structure in the flow is partially destroyed. The corresponding PDFs indicate that the primary difference in the PDFs from 2000 to $3000 \mathrm{~m}$ is that the large- $\lambda$ tail lengthens (cf. Fig. 7).

We emphasize that this behavior is not an artifact of the initial particle distribution. If a narrower initial distribution is used for $z_{0}=250 \mathrm{~m}$, a case in which most of the initial conditions yield nonchaotic trajectories, $\langle\lambda\rangle$ is even smaller, enhancing the trend in Fig. 12.

For idealized chaotic advection models, (5) is followed almost exactly (see Ngan 1997; Ngan and Shepherd 1997b). In these models it is possible to vary $\tau_{\text {rs }}$ directly when external stochasticity is introduced; moreover, feedback effects associated with stronger forcing can be largely ignored. Without stochasticity, $\langle\lambda\rangle$ scales smoothly with the perturbation amplitude $\varepsilon$, increasing roughly as a power law for large $\varepsilon$.

Another illustration of the additional complexity in the shallow-water system is given by Fig. 13. Here we see that $\langle\lambda\rangle$ drifts steadily with $\delta_{v}$. In fact $\langle\lambda\rangle \sim \delta_{v}^{1 / 2}$ : stochastic forcing in the velocity functions as if it were an additive noise term in the equations of motion (the velocity derivatives drift in time like $\delta_{v}^{1 / 2} t^{1 / 2}$ for small $\left.\delta_{v}\right)$.

\section{Concentration statistics}

Although Liapunov exponents deal only with the stretching properties of a flow, they are usually taken as a proxy for mixing, as is done above. Strictly speaking, however, the tracer field itself should be used to characterize mixing (cf. Ngan and Shepherd 1997a). We have focused upon the stretching properties of the shallow-water system because our interest is in quantitative analysis of chaotic advection rather than in mixing per se; nevertheless, it is interesting to see how the PDFs of Liapunov exponents are reflected in the PDFs of tracer concentration.

Following Pierrehumbert (1994), a continuous tracer field is constructed by associating a tracer concentration with each particle and discretizing the domain with a 


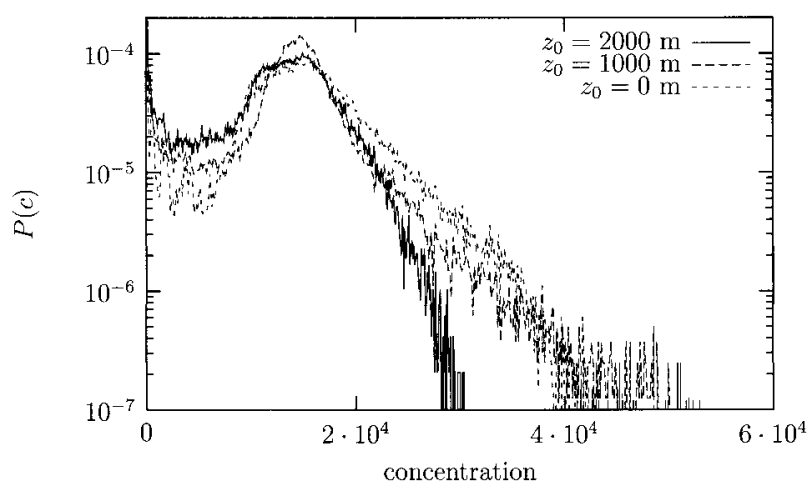

FIG. 14. $P(c)$ at 50 days for three different values of $z_{0}$. The concentration units are arbitrary.

regular latitude-longitude grid. In the calculations described below the domain $[0,2 \pi] \times\left[10^{\circ} \mathrm{N}, 60^{\circ} \mathrm{N}\right]$ is discretized using a $512 \times 128$ grid. An unavoidable limitation of the calculation is that there will be "gaps" in the PDF if the particles are not evenly distributed over the domain. This problem can be minimized if the number of grid points is reduced; however, the improvement is not significant unless a drastic reduction is imposed, in which case the PDF is not properly resolved.

In principle a well-defined PDF can be obtained by using a larger number of particles, but as quadrupling the number of particles makes little difference, this is not a practical approach. An efficient way of circumventing the problem of missing concentrations is to treat the particles as finite blobs of tracer. The concentration is assumed to decay away from the particle- the center of the blob-according to

$$
\begin{array}{llrl}
c & =c_{0} \exp \left(-\alpha r^{2}\right), & & r<r_{\text {blob }}, \\
c & =0, & r & >r_{\text {blob }},
\end{array}
$$

where $r$ is the radial separation, $r_{\text {blob }}$ is the radius of the blob, and $c_{0}$ and $\alpha$ are constants. In the numerical implementation it is convenient to take $r$ and $r_{\text {blob }}$ as integers (i.e., as grid spacings). Thus $\alpha=1 / 5^{2}$ and $r_{\text {blob }}$ $=10$ are used in the results described below. ${ }^{9}$ Particles that are initially north of $37.5^{\circ} \mathrm{N}$ are assigned a value of 300; particles initially to the south are assigned a value of 200. As a check of robustness small random fluctuations $(\sim 5 \%)$ have been introduced into the concentration values: this has no discernible effect on the shape of the PDFs.

Figure 14 shows the PDF of the concentration, $P(c)$, at 50 days for $z_{0}=0,1000$, and $2000 \mathrm{~m}$. In Fig. 14, $P(c)$ is not time independent because the use of a discrete grid amounts to the imposition of a finite tracer diffu-

\footnotetext{
${ }^{9}$ Actually, it is the cutoff in the zonal direction that is given above; the cutoff in the meridional direction is scaled to take the grid spacings into account. (Recall that the velocity field is output onto a $144 \times$ 72 grid.)
}

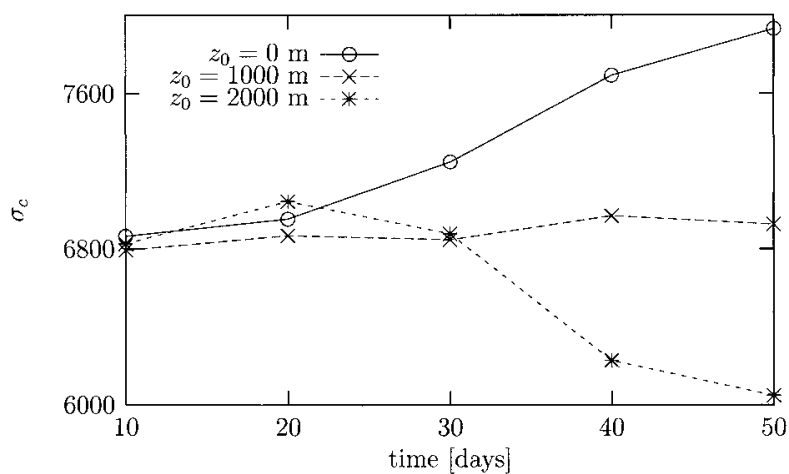

FIG. 15. Standard deviation of the concentration PDFs, $\sigma_{c}$, vs time for three different values of $z_{0}$.

sivity. The PDFs are somewhat hard to interpret because small concentrations may be overrepresented; the trick of representing each particle as a finite blob cannot alter the fact that the particles are not uniformly spaced. Nonetheless, it does appear that the PDFs are of the same general form as $P(\lambda)$. This is broadly consistent with the analysis of Chertkov et al. (1995), who show that a concentration PDF with a Gaussian core and exponential tails is expected if a delta-correlated-in-time tracer source is introduced. The addition of the deltacorrelated source simplifies the analysis and mitigates the influence of the initial tracer distribution. It is likely because of the initial conditions that the exponential tails are longer and better defined than for $P(\lambda)$ (cf. Fig. 7). The existence of long tails, be they exponential or not, is indicative of the presence of rare events; this implies that, compared with the stretching field, the tracer field is relatively inhomogeneous. Calculations with a continuous tracer field and a spatially smooth random map model yield analogous results (K. Ngan and R. T. Pierrehumbert 1999, unpublished manuscript), though the shape of the PDF is strongly influenced by the nature of the forcing, as well as by spatial resolution and tracer diffusivity. These results are consistent with those of Ching and Tsang (1997) for random advection by a spatially rough flow.

Another interesting feature of Fig. 14 is that the PDFs narrow as $z_{0}$ is increased. This can be seen very clearly in Fig. 15, which plots the standard deviation of the PDFs against time: at 50 days, $\sigma_{c}$ is much smaller for $z_{0}=2000 \mathrm{~m}$ than for $z_{0}=0$ and $1000 \mathrm{~m}$. Moreover, the time evolution of $\sigma_{c}$ closely parallels that of the analogous diagnostic, $\Delta_{K-K_{1}}$, used in the analysis of Ngan and Shepherd (1997a): development of smallscale structure at initial times ( $\sigma_{c}$ increases), is followed by coarse-grain homogenization at long times $\left(\sigma_{c}\right.$ decreases). While coarse-grain homogenization is readily apparent for $z_{0}=2000 \mathrm{~m}$, it is less prevalent than for the kinematic model because the integrations are run for fewer advective times.

Although there is little point in computing concentration PDFs for a wide range of parameters-especially 


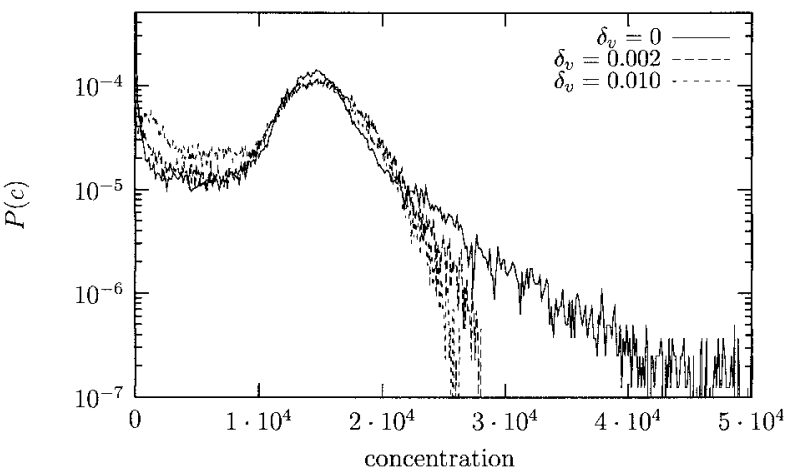

FIG. 16. $P(c)$ at 50 days for $\delta_{v}=0,0.002$, and 0.01. $z_{0}=$ $1000 \mathrm{~m}$.

since the results so obtained are nonunique: the precise results depend on how the arbitrary decay parameters $\alpha$ and $\tau_{\text {blob }}$ are defined, as well as on the initial tracer distribution-it is instructive to consider a number of cases with stochastic forcing in the velocity field. Figure 16 shows $P(c)$ at 50 days for $\delta_{v}=0,0.002$, and 0.1 . Not surprisingly, the PDFs for the stochastic cases are closer to Gaussianity than the unperturbed one. In addition there is clearly more coarse-grain homogenization for the stochastic cases ( $\sigma_{c}$ decreases), in agreement with Fig. $10 .{ }^{10}$

PDFs of concentration gradients can also be constructed. Recently there has been much interest in gradient PDFs because they provide a striking example of intermittency. While Kraichnan (1974) showed that the gradient PDF takes a lognormal form for zero diffusivity, such a PDF has not been found in experiments or simulations (Holzer and Siggia 1994); data from numerical simulations is well fit by a stretched-exponential form, and stretched exponentials have been obtained analytically for large-scale random strain (Chertkov et al. 1998). In Fig. 17, we see that the gradient PDF does take a stretched-exponential form for $z_{0}=1000 \mathrm{~m}$; there is also a "bump" in the PDF, presumably because the integration is short and inhomogeneities in the tracer field remain. The scaling extends over a larger range of values when stochasticity is introduced and deviations from exponential scaling are smaller.

\section{Calculations using isentropic winds from the Canadian Middle Atmosphere Model}

The role of the large-scale velocity field has been emphasized in the preceding sections. We now investigate whether there is the same kind of behavior in the Canadian Middle Atmosphere Model: as with the shal-

\footnotetext{
${ }^{10}$ This conclusion might appear to be at odds with results for the stochastic two-wave model of Ngan and Shepherd (1997a). Recall, however, that stochasticity in the shallow-water system has a different effect from stochasticity in a kinematic model (section 4c).
}

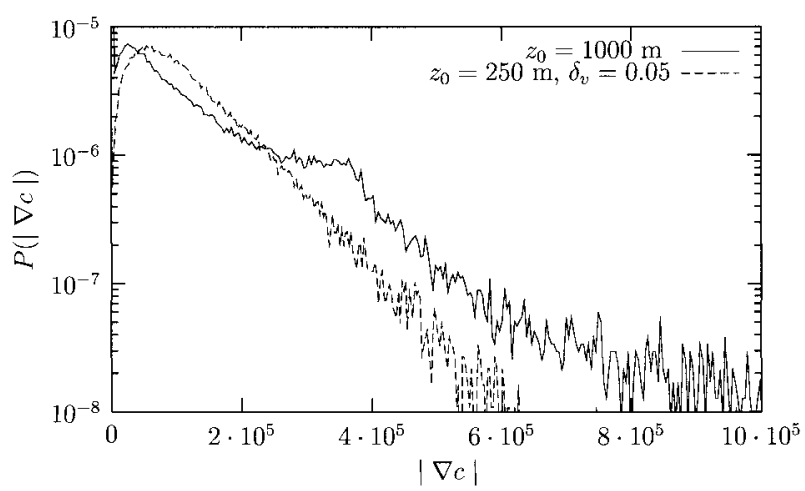

FIG. 17. $P(|\nabla c|)$ at 50 days for $z_{0}=1000 \mathrm{~m}$, and for $z_{0}=250 \mathrm{~m}$ with $\delta_{v}=0.05$. The units are arbitrary.

low-water system there is likewise large-scale structure in the velocity field of CMAM (see Part I, section 5).

Isentropic winds from 1 July to 30 July of a long simulation are used. A smaller number of particles are used than in the offline calculation; approximately 60000 points are uniformly spaced in latitude and longitude from $5^{\circ}$ to $55^{\circ} \mathrm{S}$ at $0.5^{\circ}$ intervals and from $0.02^{\circ}$ to $359.98^{\circ} \mathrm{E}$ at $0.5^{\circ}$ intervals.

\section{a. Dispersion statistics}

Figure 18 shows $\sigma_{x}^{2}$ and $\sigma_{y}^{2}$ versus time for $\theta=450$, 600 , and $1000 \mathrm{~K}$. The $\sigma_{x}^{2}$ behavior is much as before: $\sigma_{x}^{2} \sim t^{2}$, reflecting the existence of quasi-regular particle trajectories (cf. section 2). The $\sigma_{y}^{2}$ behavior, on the other hand, is somewhat different: there are large-amplitude oscillations superimposed on a time-dependent baseline. This behavior reflects the strong gravity wave signal in the CMAM winds; the fast oscillations experienced by individual particles make it difficult for a systematic trend to be identified. However, if $\sigma_{y}^{2}$ is computed for initial distributions oriented along zonal circles (not shown), then $\sigma_{y}^{2}$ once more increases and levels off with time, in agreement with the results of Bowman (1993).

In the shallow-water system the time dependence of $\sigma_{x}^{2}$ and $\sigma_{y}^{2}$ was determined by the large-scale structure of the velocity field; this is evidently still true of $\sigma_{x}^{2}$ but it is much harder to say with $\sigma_{y}^{2}$. The main value of this calculation is that it demonstrates (i) that there is no sign of diffusive tracer motion (anomalous or normal) in the zonal direction, and (ii) the limitation of the dispersion statistics as transport diagnostics.

\section{b. Liapunov exponents}

In section $4 b$ it was argued that the qualitative form of the PDFs is unchanged so long as there exists a cat's eye structure in the surf zone. This is supported by calculations with CMAM. From $\theta=450$ to $1000 \mathrm{~K}$, broad PDFs with positive skewness are found (Fig. 19). This suggests that positive skewness of the PDFs is a robust feature: the near-Gaussian PDFs obtained for 
(a)

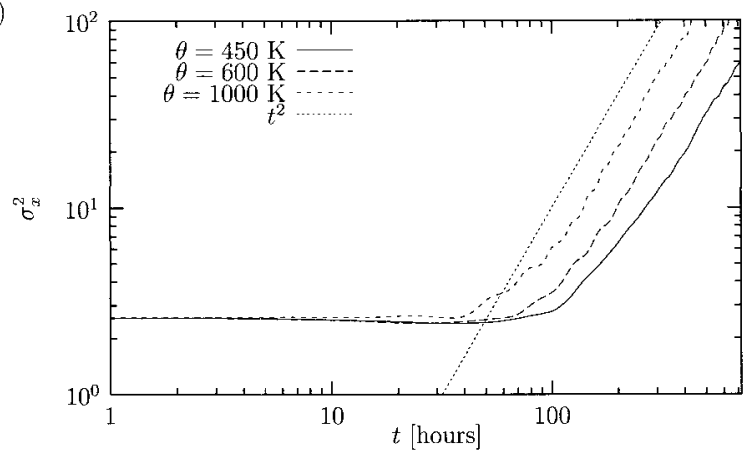

(b)

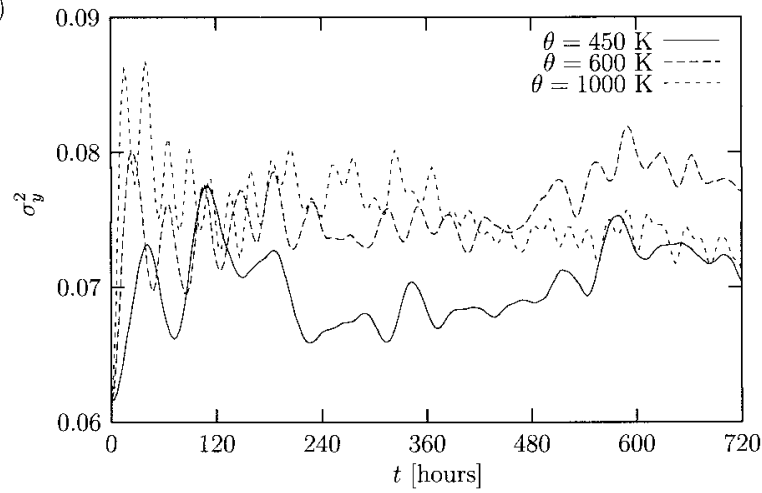

FIG. 18. Dispersion statistics from CMAM isentropic winds. (a) $\sigma_{x}^{2}$; (b) $\sigma_{y}^{2}$.

strong stochastic forcing (Fig. 10) are probably not representative of realistic flows in the lower stratosphere. A particularly striking feature of the CMAM PDFs is the increase in their broadness with height. Indeed, the PDFs for 600 and $1000 \mathrm{~K}$ are significantly broader than the shallow-water PDFs (cf. Fig. 7), even though the Lagrangian correlation time in CMAM is shorter (see Part I, Figs. 14 and 17). As with increased shear in the shallow-water system (Fig. 9), the broadening of the PDFs at higher altitude is likely related to the sampling

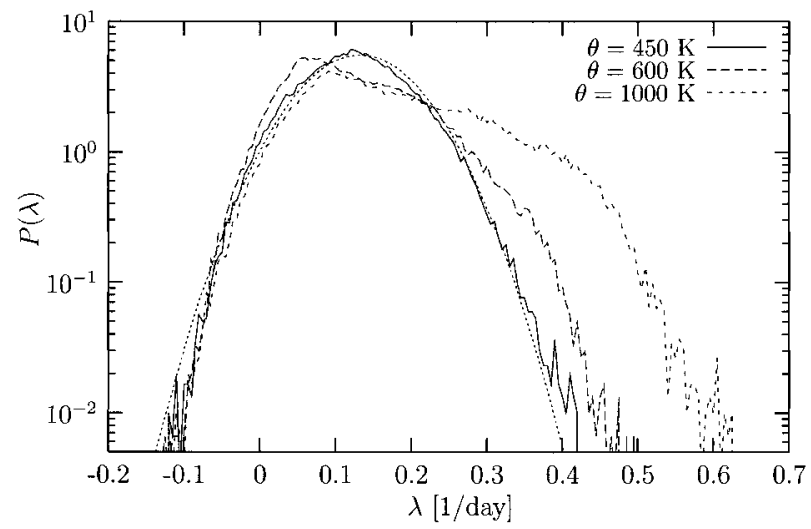

FIG. 19. $P(\lambda)$ at 30 days using isentropic winds from CMAM.

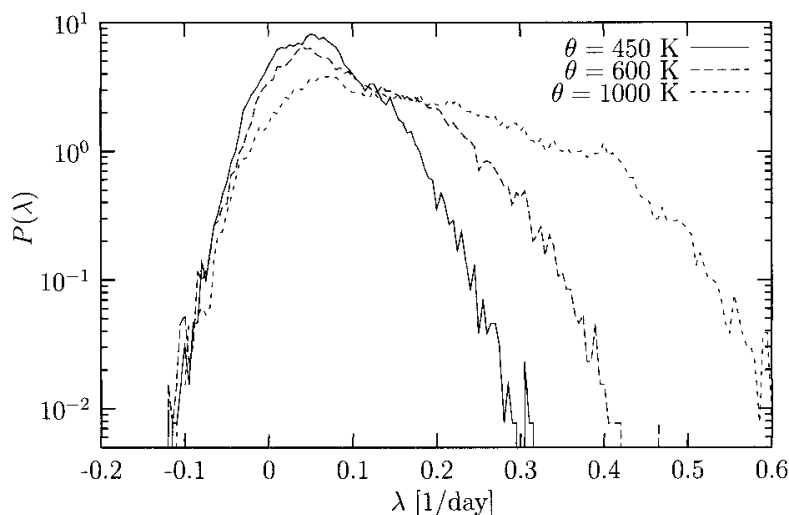

FIG. 20. $P(\lambda)$ at 30 days for an initial distribution inside the vortex. Approximately 25000 particles between $55^{\circ}$ and $80^{\circ} \mathrm{S}$ are integrated using isentropic winds from CMAM.

of a larger range of strains, the Lagrangian correlation time being approximately independent of height.

The PDFs for initial distributions inside the vortex are qualitatively similar (Fig. 20). In all cases the distributions are, as expected, shifted toward smaller $\lambda$, though the shift is greatest for $450 \mathrm{~K}$. The PDFs are also, once more, rather broad. These results are consistent with those of Pierce et al. (1994), who showed using analyzed winds and parameterized gravity waves that mixing in the polar vortex is nonnegligible.

It is also interesting to examine how the scaling of $\langle\lambda\rangle$ changes with the isentropic surface. Generally $\langle\lambda\rangle$ increases with $\theta$ (Fig. 21). In light of the shallow-water results, this is most likely due to the increase in the basic-state shear (velocities increase with height). The nonmonotonic behavior around $\theta=450 \mathrm{~K}$ could be a consequence of variations in the structure of the surf zone (see section $4 b$ ), or in the strength of the zonal jet.

\section{Discussion}

In this work we have computed a number of statistical diagnostics for the shallow-water and middle-atmosphere models. Specifically, transport has been char-

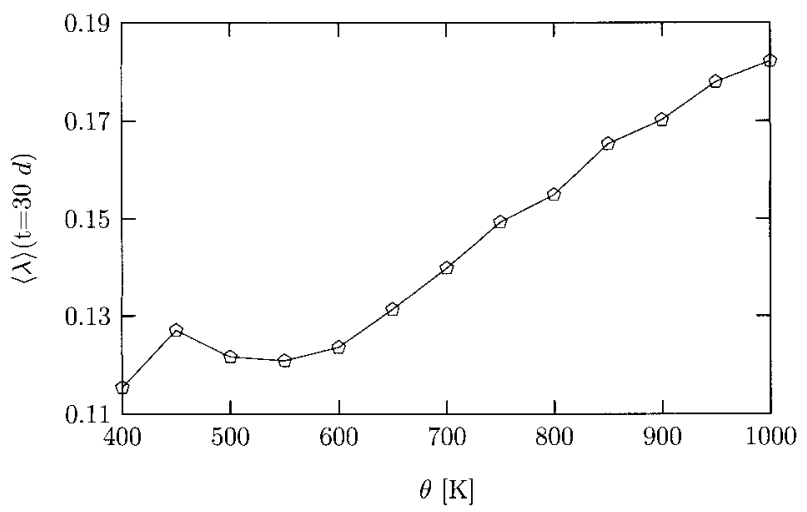

FIG. 21. $\langle\lambda\rangle(t=30 \mathrm{~d})$ vs $\theta$. 
acterized using dispersion statistics and transport fluxes, mixing with Liapunov exponents and concentration statistics. Briefly, the dispersion statistics illustrate the correspondence between the chaotic region of a dynamical system and the surf zone of the numerical models (a notion implicit in any discussion of chaotic advection in the stratosphere), the transport fluxes, the temporal and spatial variability of the stratospheric models, and the Liapunov exponents (and concentration statistics), which are the primary focus of our analysis, the differences between these models and idealized ones.

The quantitative characterization provided by these statistical diagnostics represents the main practical contribution of this work. Turning first to the Liapunov exponents, we have shown that on timescales relevant to stratospheric mixing and transport their probability distributions are broad (the standard deviation is comparable to the mean), especially for the middle-atmosphere model. This is significant because it demonstrates that, neglecting chemical and radiative effects, as well as explicit tracer diffusivity, mixing in the surf zone, on physically meaningful timescales, may be a rather inhomogeneous process. This inhomogeneity is manifested even more strikingly in the PDFs of concentration and concentration gradient, which have long non-Gaussian tails. The increased non-Gaussianity of the concentration PDF may reflect the arbitrary choice of initial conditions; it would be interesting to see how the shape of this PDF, relative to the Liapunov PDF, would be affected if a more realistic initial distribution were used and additional physics (i.e., source terms) included. Another aspect to the Liapunov PDFs is that they may prove useful in assessing the sensitivity of offline calculations to the spatial resolution of the velocity field (cf. Fig. 11); they moreover help identify situations (e.g., strong stochastic forcing) in which chaotic advection does not occur. The dispersion statistics indicate that there is minimal evidence for zonal diffusion (anomalous or normal) in the stratosphere.

The original motivation for this work, however, was not stratospheric mixing and transport per se but the relationship of chaotic advection in the stratosphere to chaotic dynamical systems and random-strain theory. The shallow-water system, not to mention the middleatmosphere model, is quite different from "classical" chaotic systems: vorticity is materially conserved (on sufficiently short timescales); fast small-scale oscillations are present; the large-scale spatial structure of the velocity field is time dependent [it evolves by an $O(1)$ amount during the course of the integration]. The rather restrictive assumptions of random-strain theory are also violated: the velocity derivatives do not all decorrelate at the same rate, for instance. Nevertheless, just as random-strain theory provides a reasonable description of chaotic dynamical systems (though some assumptions are, strictly speaking, violated as well), so too does it apply to chaotic advection in the stratosphere. One sees the emergence of a near-Gaussian probability distribu- tion of Liapunov exponents; the PDFs have positive skewness, the skewness decreasing with time, in accord with the behavior of stochastically forced Hamiltonian systems and random-strain theory. The upshot of these results is that the stratospheric models do exhibit the expected statistical behavior, though finite-time departures from asymptotic random-strain results are important. It would be useful if this statistical information could be incorporated into a model of stratospheric mixing, transport, and chemistry.

Acknowledgments. The authors thank S. Gravel and M. Roch of Recherche en Prévision Numérique (Environment Canada) for providing the shallow-water model, J. N. Koshyk for the CMAM data, R. T. Pierrehumbert and L. M. Polvani for helpful discussion and comments, and R. A. Plumb for many constructive criticisms. This work was supported by the Natural Sciences and Engineering Research Council and the Atmospheric Environment Service of Canada.

\section{REFERENCES}

Bowman, K. P., 1993: Large-scale isentropic mixing properties of the Antarctic polar vortex from analyzed winds. J. Geophys. Res., 98, $23013-23027$.

Chertkov, M., G. Falkovich, I. Kolokolov, and I. Lebedev, 1995: Statistics of a passive scalar advected by a large-scale two-dimensional velocity field: Analytic solution. Phys. Rev. E, 51, $5609-5627$.

,-- , and -1998 : Intermittent dissipation of a passive scalar in turbulence. Phys. Rev. Lett., 80, 2121-2124.

Ching, E. S. C., and Y. K. Tsang, 1997: Passive scalar conditional statistics in a model of random advection. Phys. Fluids, 9, 13531361.

Crisanti, A., and A. Vulpiani, 1993: On the effects of noise and drift on diffusion in fluids. J. Stat. Phys., 70, 197-211.

Duan, J., and S. Wiggins, 1996: Fluid exchange across a meandering jet with quasiperiodic variability. J. Phys. Oceanogr., 26, 11761188.

Geist, K., U. Parlitz, and W. Lauterborn, 1990: Comparison of different methods for computing Lyapunov exponents. Prog. Theor. Phys., 83, 875-893.

Haynes, P. H., and J. Anglade, 1997: The vertical-scale cascade in atmospheric tracers due to large-scale differential advection. $J$. Atmos. Sci., 54, 1121-1136.

Holzer, M., and E. D. Siggia, 1994: Turbulent mixing of a passive scalar. Phys. Fluids, 6, 1820-1837.

Horita, T., H. Hata, R. Ishizaki, and H. Mori, 1990: Long-time correlations and expansion-rate spectra of chaos in Hamiltonian systems. Prog. Theor. Phys., 83, 1065-1070.

Juckes, M. N., and M. E. McIntyre, 1987: A high-resolution onelayer model of breaking planetary waves in the stratosphere. Nature, 328, 590-596.

Kraichnan, R. H., 1974: Convection of a passive scalar by a quasiuniform random straining field. J. Fluid Mech., 64, 737-762.

Lichtenberg, A. J., and M. A. Lieberman, 1992: Regular and Chaotic Dynamics. 2d ed. Springer, $692 \mathrm{pp}$.

Mezić, I., and S. Wiggins, 1994: On the dynamical origin of asymptotic $t^{2}$ dispersion of a nondiffusive tracer in incompressible laminar flows. Phys. Fluids, 6, 2227-2229.

Ngan, K., 1997: Chaotic advection in the stratosphere. Ph.D. thesis, University of Toronto, $179 \mathrm{pp}$.

, and T. G. Shepherd, 1997a: Chaotic mixing and transport in Rossby-wave critical layers. J. Fluid Mech., 334, 315-351. 

stratosphere. Preprints, 11th Conf. on Atmospheric and Oceanic Fluid Dynamics, Tacoma, WA, Amer. Meteor. Soc., 50-53.

- and - 1999: A closer look at chaotic advection in the stratosphere. Part I: Geometric structure. J. Atmos. Sci., 56, 4134-4152.

Pierce, R. B., T. D. Fairlie, W. L. Grose, R. Swinbank, and A. O'Neill, 1994: Mixing processes within the polar night jet. J. Atmos. Sci., 51, 2957-2972.

Pierrehumbert, R. T., 1994: Tracer microstructure in the large-eddy dominated regime. Chaos Applied to Fluid Mixing, H. Aref and M. S. El Naschie, Eds., Pergamon, 347-366.

Polvani, L. M., D. W. Waugh, and R. A. Plumb, 1995: On the subtropical edge of the stratospheric surf zone. J. Atmos. Sci., 52, 1288-1309.

Rom-Kedar, V., A. Leonard, and S. Wiggins, 1990: An analytical study of transport, mixing and chaos in an unsteady vortical flow. J. Fluid Mech., 246, 347-394.

Schoeberl, M. R., and P. A. Newman, 1995: A multiple-level trajec- tory analysis of vortex filaments. J. Geophys. Res., 100, 25 80125815 .

Shlesinger, M. F., G. M. Zaslavsky, and J. Klafter, 1993: Strange kinetics. Nature, 363, 31-37.

Sobel, A. H., R. A. Plumb, and D. W. Waugh, 1997: Methods of calculating transport across the polar vortex edge. J. Atmos. Sci., 54, 2241-2260.

Tabor, M., and I. Klapper, 1994: Stretching and alignment in chaotic flows. Chaos Applied to Fluid Mixing, H. Aref and M. S. El Naschie, Eds., Pergamon, 287-311.

Városi, F., T. M. Antonsen, and E. Ott, 1991: The spectrum of fractal dimensions of passively convected scalar gradients in chaotic fluid flows. Phys. Fluids A, 3, 1017-1028.

von Bremen, H. F., F. E. Udwadia, and W. Proskurowski, 1997: An efficient QR based method for the computation of Lyapunov exponents. Physica D, 101, 1-16.

Waugh, D. W., and R. A. Plumb, 1994: Contour advection with surgery: A technique for investigating fine-scale structure in tracer transport. J. Atmos. Sci., 51, 530-540.

Wiggins, S., 1992: Chaotic Transport in Dynamical Systems. Springer, $301 \mathrm{pp}$. 